Revista de Investigación Educativa 28

enero-junio, 2019 | ISSN 1870-5308 | Xalapa, Veracruz

Instituto de Investigaciones en Educación | Universidad Veracruzana

\title{
Representaciones sociales de las prácticas científicas en los textos escolares de ciencias naturales utilizados en escuelas chilenas sostenidas con fondos públicos
}

\author{
Social representations of scientific practices in primary textbooks \\ of sciences used in chilean schools supported by public funds
}

\author{
Jennifer Andrea Venegas Espinoza \\ Recibido: 07 de mayo de 2018 \\ Aceptado: 15 de diciembre de 2018 \\ DOI: https://doi.org/10.25009/cpue.v0i28.2599
}

La investigación pretendió explorar las representaciones sociales que se realizan del quehacer científico en los textos del estudiante para la asignatura de ciencias naturales, utilizados en enseñanza básica en escuelas sostenidas con fondos públicos de Chile, por medio de los conceptos de Ciencia en acción y Ciencia elaborada desarrollados por B. Latour. Los textos analizados corresponden a los entregados y distribuidos de manera gratuita por el Ministerio de Educación a estudiantes de primaria. Se ha podido evidenciar que las prácticas presentadas varían por curso y por unidad; éstas tienden a reproducir representaciones asociadas a una ciencia elaborada, ya que se presentan prácticas propias de una ciencia absoluta, con saberes imposibles de ser modificados y muchas veces carecen de contexto y de historia. Los estudiantes deben corroborar saberes previamente establecidos, por lo tanto, se transmite que la ciencia es meramente comprobatoria y no explicativa.

Palabras clave: Libros de texto gratuitos; educación primaria; representación social; educación científica; Chile.

\footnotetext{
a Doctoranda en Estudios de Género. Universidad de Barcelona, España.

凶 jennifer.venegas.es@gmail.com
} 
The aim of the present investigation was to explore the social representations that are made of the scientific practices in the students' texts for the curricular subject of natural sciences used in schools supported by public funds of Chile, through the concepts of Science in action and Elaborated science developed by B. Latour. The texts analyzed correspond to those delivered and distributed free of charge by the Ministry of Education to primary students. It has been possible to demonstrate that the practices presented, vary as much by grade as by unit, these tend to reproduce representations associated to an elaborated science, since they present own practices of an absolute science, with knowledge impossible to be modified, this often lacks context and history. The students must corroborate previously established knowledge, therefore it is transmitted that the science is merely demostrative and not explanatory.

Keywords: Free textbooks; primary education; social representation; science education; Chile.

\title{
Representaciones sociales de las prácticas científicas en los textos escolares de ciencias naturales utilizados en escuelas chilenas sostenidas con fondos públicos
}

\author{
Social representations of scientific practices in primary textbooks \\ of sciences used in chilean schools supported by public funds
}

\section{Introducción}

$\mathrm{L}$ a presente investigación pretendió explorar las diferentes representaciones sociales que se realizan del quehacer científico a través de lo presentado en los textos del estudiante, entregados de manera gratuita por el Ministerio de Educación (MINEDUC) a cada uno de los colegios municipales y particulares subvencionados de Chile, a la luz de los conceptos de ciencia en acción y ciencia elaborada, de Bruno Latour (1992; 2001). 
Los textos utilizados se distribuyen anualmente y en particular éstos han sido diseñados bajo el alero de las Bases Curriculares 2012, marco curricular que procura desarrollar competencias científicas en cada uno de los estudiantes chilenos.

Los programas de estudio en nuestro país se acompañan de una serie de manuales, entregados tanto a docentes como a estudiantes. Estos libros entregan visiones oficiales de lo que se entiende por ciencia y por práctica científica. Al exponernos a ellos, estos nos interpelan y nos van construyendo mediante discursos de habilitación y supresión (Yáñez, 2010). Ofrecen imágenes y prácticas representacionales establecidas, incorporándose éstas en nuestros sistemas de creencia y de acción.

\section{Ciencia en Acción versus Ciencia Elaborada}

Bruno Latour y otros autores indican que la ciencia y los procesos de investigación científica, corresponden a construcciones propias de un proceso social (Ziman, 2003). Se establece que si un fenómeno es considerado como algo construido científicamente, es porque tanto el hecho, los instrumentos y los investigadores han pasado por un proceso de interpretación, interacción y difusión en un entramado social, constituido por un sistema de redes (Latour, 2001).

Pero de esta ciencia construida, ¿qué es lo que llega a nosotros? Latour (1992), en Ciencia en Acción indica que el conocimiento construido se muestra ya elaborado, se nos presentan conceptos, máquinas y técnicas, todas ellas dentro de una gran caja negra. Por lo tanto, se desprenden dos posibles representaciones del proceso de investigación científica, una que muestra una ciencia en proceso de elaboración o en acción versus otra que nos muestra una ciencia acabada o elaborada. La imagen de ciencia acabada; recibe este nombre por presentar a la ciencia como algo resuelto; se ofrece a la comunidad una serie de elementos ya definidos, sin permitir la comprensión en profundidad del proceso que permitió que se generara. Lo único que sabemos es cómo funcionan externamente esos objetos (Latour, 1983). Con esto, el conocimiento que se posee de la naturaleza misma de la ciencia es ingenuo y poco real. Por el contrario, la ciencia en acción representa a la investigación como un proceso, el cual se caracteriza por reconocer la participación trascendental que tiene cada uno de los actantes en la misma; en este proceso el actor humano vive y desarrolla un modo particular de pensar, siendo la experimentación un acto de reconocimiento y construcción. 


\section{Representaciones sociales}

Para este estudio en particular, la característica de las representaciones sociales que cobra mayor relevancia es la que se refiere a las representaciones como un tipo de conocimiento socialmente elaborado y además compartido, con una orientación más bien pragmática y enfocada a la construcción de una realidad común en un conjunto social; por esto es entendido como el saber del sentido común (Domínguez, 2009).

Moscovici $(1984 ; 1988 ; 2000)$ comprende el concepto como un sistema complejo de ideas, prácticas y valores, en el cual se establece un sistema de referencia que orienta a los sujetos en su mundo material y social, entregándoles así un "código" que permite el intercambio y la participación del sujeto en un grupo social.

Se puede indicar, por tanto que, la representación social nos otorga una forma de pensamiento social, el cual surge en un contexto de relaciones cotidianas y de acciones sociales propias de un grupo en un momento y en una situación particular.

Un texto escolar, por ejemplo, se transforma en una fuente de información sustancial a la hora de construir determinadas representaciones sociales. La escuela y el aula, en particular, son un espacio de difusión y construcción de las mismas. Ante esto, la representación social participa tanto en el desarrollo individual como colectivo de los sujetos y en la definición de las identidades personales y colectivas. Al poseer un componente cognitivo, la representación social permite a los sujetos una pertenencia con implicancias afectivas y normativas en los diferentes espacios sociales (Gunter, 2002).

\section{Diseño de investigación y metodología}

La investigación tiene un alcance exploratorio-descriptivo y se ocupó de los libros de texto para el estudiante, en particular los de la asignatura de ciencias naturales, tratando, a partir de estos de describir y analizar las representaciones sociales que se realizan del quehacer científico. Se considera que los libros de texto representan un vehículo de socialización, ofrecen una determinada visión en relación con ciertos temas, ya que se encuentran en concordancia con el proyecto político y social de quienes los producen (Yáñez, 2010).

Se escogieron, de manera específica, los textos de primero a sexto de primaria, ya que estos han pasado por una reestructuración curricular (en 2012) y en la actualidad 
se encuentran en vigencia. Además, los estudiantes que reciben estos textos se encuentran en una etapa de desarrollo durante la cual se construyen fundamentalmente las ideas de mundo de las personas (Devés, 2007; Organización de las Naciones Unidas para la Educación, la Ciencia y la Cultura, 2000), por lo que es vital conocer las representaciones que los textos "traen" consigo.

Se establecieron como unidad de análisis las acciones que se les propone realizar a los estudiantes dentro de los textos de estudio; estas acciones (prácticas y reflexiones) permitieron posteriormente interpretar qué tipo de representación de las ciencias naturales está siendo construida por dichos documentos.

Para poder realizar el análisis se establecieron cinco categorías de codificación en el programa Nvivo 8, las cuales se presentan en la Tabla 1.

\section{Tabla 1. Categorías teóricas establecidas para la codificación}

\begin{tabular}{|c|c|}
\hline Categorías & Descripción \\
\hline $\begin{array}{l}\text { Acciones o prácticas } \\
\text { propias del quehacer } \\
\text { científico. }\end{array}$ & $\begin{array}{l}\text { Acciones que promueven prácticas propias del quehacer científico; } \\
\text { éstas desafían a los estudiantes a desarrollar actividades como: } \\
\text { problematizar, hipotetizar, diseñar modelos de contrastación, concluir } \\
\text { con base en la evidencia, etc. } \\
\text { Evidencian un proceso de construcción científica mediante procesos } \\
\text { propios de las prácticas científicas (Driver, 2000; Harlen, s.f.; Haury, 1993; } \\
\text { Holbrook \& Raminknae, 2007; Latour, 1992, 2001). }\end{array}$ \\
\hline $\begin{array}{l}\text { Reflexiones que } \\
\text { posibilitan la } \\
\text { comprensión de las } \\
\text { prácticas científicas. }\end{array}$ & $\begin{array}{l}\text { Acciones que promueven reflexiones o cuestionamientos de las } \\
\text { prácticas científicas; éstas permiten comprender en profundidad los } \\
\text { procesos de construcción de los saberes científicos } \\
\text { (Driver, 2000; Latour, 2001; Pugh, 2009). }\end{array}$ \\
\hline $\begin{array}{l}\text { Acciones que tratan } \\
\text { conceptos de manera } \\
\text { elaborada o dentro de } \\
\text { una caja negra. }\end{array}$ & $\begin{array}{l}\text { Acciones que tratan conceptos o herramientas de manera } \\
\text { desnaturalizada, sin cuestionar los procesos que los produjeron. Se } \\
\text { centran en el conocimiento ya elaborado como producto (Cofré, } \\
\text { Camacho, Galaz, Jiménez, Santibáñez \& Vergara, 2010; Hughes, 2000; } \\
\text { Latour, 1992, 2001). }\end{array}$ \\
\hline $\begin{array}{l}\text { Acciones comunes a } \\
\text { cualquier disciplina. }\end{array}$ & $\begin{array}{l}\text { Acciones o prácticas que se realizan desde cualquier disciplina, no } \\
\text { teniendo un vínculo directo con las prácticas científicas. Por ejemplo: } \\
\text { unir, ubicar, subrayar, leer. }\end{array}$ \\
\hline
\end{tabular}




\begin{tabular}{ll}
\hline \multicolumn{1}{c}{ Categorías } & \multicolumn{1}{c}{ Descripción } \\
\hline $\begin{array}{l}\text { Acciones o prácticas } \\
\text { ingenuas. }\end{array}$ & $\begin{array}{l}\text { Acciones o prácticas vinculadas a situaciones que implican el seguir } \\
\text { pasos, el replicar modelos ya establecidos. Éstas promueven un saber } \\
\text { ingenuo de las prácticas científicas, no permiten comprender en } \\
\text { profundidad las características del proceso de construcción científica } \\
\text { (Ledesma, 2005; Bunge, 1960). }\end{array}$ \\
\hline
\end{tabular}

Fuente: Elaboración propia.

\section{Resultados y análisis de datos}

\subsection{Resultados}

Del total de acciones analizadas en los textos de estudio, se observó que las acciones menos presentadas son las vinculadas con los quehaceres propios de las prácticas científicas, así como también las acciones que incitan la reflexión profunda en relación con las mismas (Tabla 2).

Tabla 2. Frecuencia de aparición de acciones por categoría de análisis

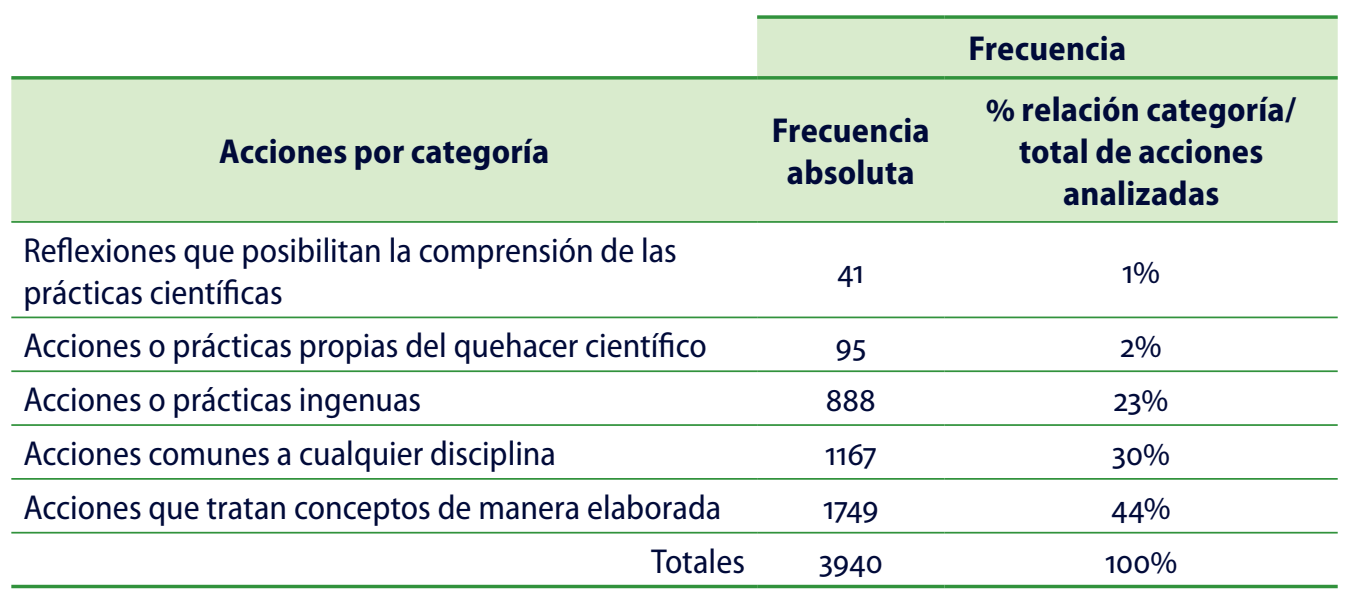

Fuente: Elaboración propia. 


\subsubsection{Comportamiento de la categoría en cada uno de los manuales}

En el siguiente apartado se muestra la frecuencia de aparición de cada una de las categorías teóricas presentes en los diferentes textos analizados. Para la presentación y análisis de los mismos se realizó una categorización por curso o nivel de estudio.

\subsubsection{Acciones-Prácticas propias del quehacer científico}

Este tipo de acciones se encuentra presente en los textos de estudio, pero en muy baja cantidad (Tabla 3).

\section{Tabla 3. Acciones prácticas propias del quehacer científico}

\begin{tabular}{lcc}
\hline Recursos & Frecuencia absoluta & $\%$ \\
\hline Texto $1^{\circ}$ básico & 10 & $11 \%$ \\
\hline Texto $2^{\circ}$ básico & 14 & $15 \%$ \\
\hline Texto $3^{\circ}$ básico & 30 & $32 \%$ \\
\hline Texto $4^{\circ}$ básico & 28 & $29 \%$ \\
\hline Texto $5^{\circ}$ básico & 12 & $13 \%$ \\
\hline Texto $6^{\circ}$ básico & 1 & $1 \%$ \\
\hline Totales & 95 & $100 \%$ \\
\hline
\end{tabular}

Fuente: Elaboración propia.

Las acciones propuestas incluidas en los textos de cursos inferiores (primer y segundo año básico) presentan actividades que se relacionan con el poder concluir con base en resultados o en evidencia empírica vinculada con alguna experiencia práctica o con alguna experiencia previa. Se puede indicar también que las acciones propuestas brindan gran importancia a la comprobación. Lo anterior se puede evidenciar en la siguiente cita extraída del texto de primer año básico: “¿Qué necesidad de las plantas comprobaste en este experimento? ¿Qué más necesitan las plantas para vivir?” (MINEDUC, 2012a, p. 21).

Ahora bien, en los textos de tercer y cuarto básico se aprecian los porcentajes más altos en esta categoría. A continuación se presentan citas extraídas desde el texto de cuarto año básico: 
4. Diseña tu prueba. Dibuja cómo armarás tu prueba.

5. Enumera tus pasos en el orden en que los realizarás.

\section{Haz tu prueba.}

6. Sigue los pasos que escribiste.

7. Asegúrate de escribir tus observaciones en la tabla. Lo que se muestra más abajo.

\section{Reúne y anota tus datos.}

8. Completa la tabla.

\section{Interpreta tus datos.}

9. Usa tus datos para hacer gráficos de barras.

10. Compara lo que viste en los dos gráficos.

\section{Plantea tu conclusión.}

11. Comunica tu conclusión. Compara tu hipótesis con tus resultados. Luego, compártelos con los demás y escribe tu conclusión. (MINEDUC, 2012c, p. 115, 116)

En los textos de quinto y sexto año básico, las acciones incluidas se relacionan con experiencias que permiten concluir y analizar resultados de investigaciones desarrolladas por otros. Además, presentan limitados casos en donde se permite desarrollar investigaciones abiertas o guiadas por los mismos estudiantes. Estas experiencias al ser aisladas limitarían el proceso de construcción social y cognitiva asociado a representaciones sociales más bien propias de la ciencia en acción.

Lo discutido anteriormente se puede apreciar en la siguiente cita:

Planteen tres conclusiones relacionadas con el análisis de los resultados.

¿Qué podrían investigar respecto de la relación que existe entre el ejercicio físico y la cantidad de respiraciones y latidos cardíacos? Elaboren una pregunta de investigación válida para hacer una investigación científica. (MINEDUC, 2013a, p. 64)

\subsubsection{Acciones-Reflexiones que posibilitan la comprensión de las prácticas científicas}

En primer lugar, cabe mencionar que esta categoría pretende incluir a toda aquella acción que promueva una comprensión de los procesos de construcción del conocimiento científico, ahondando en los procesos que posibilitaron dicha construcción (Tabla 4). 


\section{Tabla 4. Acciones-Reflexiones que posibilitan la comprensión de las prácticas científicas}

\begin{tabular}{lcc}
\hline \multicolumn{1}{c}{ Recursos } & Frecuencia absoluta & $\%$ \\
\hline Texto $1^{\circ}$ básico & 0 & $0 \%$ \\
\hline Texto $2^{\circ}$ básico & 0 & $0 \%$ \\
\hline Texto $3^{\circ}$ básico & 9 & $22 \%$ \\
\hline Texto $4^{\circ}$ básico & 10 & $24 \%$ \\
\hline Texto $5^{\circ}$ básico & 14 & $34 \%$ \\
\hline Texto $6^{\circ}$ básico & 8 & $20 \%$ \\
\hline Totales & 41 & $100 \%$ \\
\hline
\end{tabular}

Fuente: Elaboración propia.

En los textos de primer y segundo año básico, no se identificaron acciones que inciten a la reflexión en torno a la construcción del conocimiento científico.

En los textos de $3^{\circ}$ y $4^{\circ}$ año básico, se aprecia un mayor número de actividades que promueven reflexiones en relación con la realización de las prácticas científicas (Tabla 4). Ambos documentos plantean ítems que llevan por nombre "Qué es la Ciencia” " “Cómo es la Ciencia”.

Esto puede evidenciarse en las siguientes citas:

"Por qué crees que es importante usar modelos en ciencia, especialmente en astronomía?” (MINEDUC, 2012b, p. 138)

"Este científico examina una muestra de hielo proveniente de la Antártica. ¿Crees que otros científicos puedan repetir este experimento? ¿Por qué?” (MINEDUC, 2012c, p. 46).

“iTres pruebas son suficiente evidencia para establecer patrones?" (MINEDUC, 2012c, p.68).

"Los científicos que elaboraron el modelo de auto de la imagen buscaron información en un blog de Internet. ¿Crees que podrían usar esa información para resolver un problema científico que hayan definido?" (MINEDUC, 2012c, p. 90).

Este tipo de reflexiones incita a la comprensión de los procesos de investigación científica, permite trabajar a temprana edad la identificación y el reconocimiento de ciertos aspectos claves vinculados con el quehacer científico. Por ejemplo, pregun- 
tarse por la construcción de modelos y no tan sólo incitar la memorización de los mismos. Además, este tipo de reflexiones tiene el potencial de ahondar no tan sólo en la comprensión de un concepto aislado, sino que, por el contrario, permite comprender cualquier fenómeno que ha sido construido bajo las bases del pensamiento científico.

Las dos categorías mencionadas con anterioridad, relacionadas con la "reflexividad" y las "prácticas propias del quehacer científico", por la manera en que tratan a las prácticas y al conocimiento científico, de acuerdo con la propuesta que realiza esta investigación, cooperarían en la construcción de una representación social más bien de una "ciencia en acción", una ciencia en proceso, una ciencia que se construye.

A través de estas acciones podemos comprender a la ciencia en su naturaleza misma, evidenciamos procesos, redes y engranajes, comprendemos también a los sujetos humanos y no humanos, evidenciamos aspectos del contexto y de los contenidos.

2.1.1.3 Acciones que tratan conceptos elaborados (CCN)

Dentro de esta categoría se incluyeron todas las acciones que tratan conceptos, hechos o herramientas científicas, sin cuestionar los procesos que los produjeron, centrándose en el conocimiento ya elaborado como un producto. Para esta categoría se identificó un gran número de acciones en todos los textos analizados (Tabla 5).

\section{Tabla 5. Acciones que tratan conceptos elaborados}

\begin{tabular}{lcc}
\hline \multicolumn{1}{c}{ Recursos } & Frecuencia absoluta & $\%$ \\
\hline Texto $1^{\circ}$ básico & 270 & $15 \%$ \\
\hline Texto $^{\circ}$ básico & 254 & $14,5 \%$ \\
\hline Texto $^{\circ}$ básico & 255 & $14,5 \%$ \\
\hline Texto $^{\circ}$ básico & 350 & $20 \%$ \\
\hline Texto $^{\circ}$ básico & 308 & $18 \%$ \\
\hline Texto $6^{\circ}$ básico & 312 & $18 \%$ \\
\hline Totales & 1749 & $100 \%$ \\
\hline
\end{tabular}

Fuente: Elaboración propia. 
Las preguntas o acciones presentadas en los manuales permiten identificar características de determinados fenómenos así como analizarlos, pero no permiten comprender el origen de los mismos o las circunstancias en las que se produjeron, obviando las humanidades científicas. Con esto podrían estar entregando una visión de una ciencia más bien acabada y absoluta, ya que presentan fenómenos resueltos, que podrían o deberían comprobarse en las salas de clases, a través, por ejemplo, de experiencias prácticas que nos presentan a la ciencia como un recetario aislado y desprovisto de contexto y humanidades.

Cada uno de los aspectos aquí expuestos se aprecia en las siguientes citas:

Comenten en grupo:

a. Si es de día en un lado de la Tierra, ¿en qué etapa del ciclo diario se encuentra la otra mitad?

b. ¿Puede ser de día en toda la Tierra al mismo tiempo? (MINEDUC, 2012a, p. 129)

Completa las oraciones: a. El agua puede algunas sustancias como el azúcar y la . b. En los océanos y mares existe disuelta en el agua. Une el fenómeno atmosférico con su descripción. Une cada instrumento con el fenómeno que mide. (MINEDUC, 2013b, p. 129)

“¿Qué necesitan las plantas para vivir?” (MINEDUC, 2012b, p. 12).

“¿Cómo hacen estos árboles para vivir y crecer junto al agua?” (MINEdUC, 2012b, p. 13).

“Cómo usan las plantas sus hojas para producir alimento?" (MINEDUC, 2012b, p. 16).

Se aprecian preguntas que tratan conceptos al "desnudo", sin historia, sin creadores o sin espacios de creación. Se centralizan en las características y propiedades del fenómeno y de manera preponderante se centran en el estudio memorístico del mismo, esperando la reproducción de sus características por parte de los estudiantes.

\subsubsection{Acciones comunes a cualquier disciplina (PC)}

En esta categoría se incluyeron todas las acciones que se pueden desarrollar en cualquier otra disciplina o subsector de aprendizaje (artes visuales, matemáticas, lenguaje y comunicación, historia, geografía y ciencias sociales, educación física, etc.) y que además no representan necesariamente una práctica propia del quehacer científico. Estas acciones se encuentran presentes en todos los textos analizados y en cada uno de ellos se exhiben en gran cantidad, más de cien veces por texto (Tabla 6). 
Tabla 6. Acciones-prácticas comunes a cualquier disciplina

\begin{tabular}{lcc}
\hline \multicolumn{1}{c}{ Recursos } & Frecuencia absoluta & $\%$ \\
\hline Texto $1^{\circ}$ básico & 228 & $20 \%$ \\
\hline Texto $2^{\circ}$ básico & 154 & $13 \%$ \\
\hline Texto $3^{\circ}$ básico & 229 & $20 \%$ \\
\hline Texto $4^{\circ}$ básico & 230 & $20 \%$ \\
\hline Texto $5^{\circ}$ básico & 150 & $13 \%$ \\
\hline Texto $6^{\circ}$ básico & 176 & $15 \%$ \\
\hline Totales & 1167 & $100 \%$ \\
\hline
\end{tabular}

Fuente: Elaboración propia.

Las acciones solicitadas van desde unir palabras clave con determinadas imágenes, hasta crear mapas conceptuales, luego de desarrollar una lectura comprensiva de un determinado texto. Tal situación se evidencia en las siguientes citas:

"Pinta el recuadro cuando la respuesta es SÎ́" (MINEDUC, 2012a, p. 17).

"Une con una línea cada estímulo con la reacción” (MINEDUC, 2012a, p. 19).

"Recorta y pega el rompecabezas" (MINEDUC, 2012a, p. 137).

"Lee los elementos del texto que aparecen en la siguiente tabla. Busca los elementos del texto en las páginas del libro que se muestran arriba. Escribe una pista que dé cada elemento sobre el contenido" (MINEDUC, 2012b, p. 15).

"Elabora un mapa conceptual o un cuadro sinóptico que te permita resumir los aspectos más importantes de esta unidad. Puedes utilizar los siguientes conceptos" (MINEDUC, 2013C, p. 41).

\subsubsection{Acciones-Prácticas ingenuas}

En esta categoría se incluyeron todas aquellas prácticas que podrían entenderse como científicas, pero que nos entregan una imagen ingenua de lo que ésta es y representa. Dentro de estas acciones se encuentra, por ejemplo, solicitar a los estudiantes el seguir pasos previamente establecidos por otros para comprobar una verdad ya dada, así como también el replicar modelos experimentales.

Esta categoría se encontraba altamente presente en los textos de estudio analizados, encontrándose en cada uno de ellos en más de cien ocasiones por nivel (Tabla 7). 
Tabla 7. Acciones-Prácticas ingenuas

\begin{tabular}{lcc}
\hline \multicolumn{1}{c}{ Recursos } & Frecuencia absoluta & $\%$ \\
\hline Texto $1^{\circ}$ básico & 141 & $16 \%$ \\
\hline Texto $2^{\circ}$ básico & 112 & $13 \%$ \\
\hline Texto $3^{\circ}$ básico & 144 & $16 \%$ \\
\hline Texto $4^{\circ}$ básico & 181 & $20 \%$ \\
\hline Texto $5^{\circ}$ básico & 180 & $20 \%$ \\
\hline Texto $6^{\circ}$ básico & 130 & $15 \%$ \\
\hline Totales & 888 & $100 \%$ \\
\hline
\end{tabular}

Fuente: Elaboración propia.

En los textos de primero y segundo año básico se presentan actividades con el título de investigaciones científicas, en las que se le solicita a las estudiantes recolectar materiales, así como el seguir una serie de pasos para verificar algo que está asumido como verdad desde el comienzo de la actividad.

Tales aspectos quedan evidenciados en las siguientes citas:

Experimento con mi sentido del olfato: ¿Qué necesito? Venda, pocillos pequeños, alimentos para degustar. ¿Qué debo hacer? Probar alimentos con los ojos vendados. a. Recorta la tabla de la página 87 y pégala en tu cuaderno para registrar los resultados del experimento. b. Marca con un $\sqrt{ }$. ¿Cómo tuviste más aciertos sobre lo que estabas comiendo? Nariz tapada. Nariz destapada. (MINEDUC, 2012a, p. 61)

Observo y describo: ¿Qué necesito? Guantes de goma, papel absorbente, tijeras, un chorito, almeja o macha. ¿Qué debo hacer? 1. Observa atentamente y responde en tu cuaderno. a. ¿Cómo es la concha del animal?, ¿es dura o blanda? b. ¿Qué color tiene? c. Abran las conchas, ¿qué hay en su interior? d. Toquen su cuerpo, ¿cómo es?, ¿es duro o blando? e. Observen su interior, ¿tiene huesos. (MINEDUC, 2013b, p. 24)

Estas actividades centran el foco de la atención en la recolección de variados materiales y en la "obediencia" a la hora de seguir ciertos pasos, estableciendo a priori que si algo llegara a fallar en el trabajo general o en los resultados del mismo, es por no seguir las instrucciones que fueron establecidas con anterioridad, por un grupo de sujetos ajenos a nuestra realidad. 
En los textos de tercer y cuarto año básico, también se aprecia un alto número de actividades que promueven saberes ingenuos respecto a la investigación científica.

En estos textos el mayor número de actividades se centra en la realización de "investigaciones" en las que los estudiantes deben cumplir al pie de la letra, cual receta de cocina, una serie de pasos para poder llegar a la verdad dada por "los científicos".

Tal aspecto queda de manifiesto en la siguiente cita representativa:

Investígalo!

\section{¿Cómo influye la pendiente de un arroyo en la velocidad de su corriente?}

Materiales: transportador, probeta, manguera de plástico transparente, embudo, vaso plástico, agua, cronómetro, cinta adhesiva de papel.

Procedimiento

1 Haz un modelo de un arroyo. Pídele a un compañero que sostenga en alto un pedazo de manguera. Establece el ángulo del arroyo en $10^{\circ}$. Coloca un vaso en el extremo inferior del arroyo.

2 Mide $50 \mathrm{ml}$ de agua en una probeta.

3 Inserta un embudo en la parte superior de la manguera. Haz funcionar el cronómetro cuando viertas el agua en la manguera. Detén el cronómetro cuando toda el agua haya fluido dentro del vaso. Anota el tiempo.

4 Cambia el ángulo del arroyo a $25^{\circ}, 40^{\circ}$ y $55^{\circ}$, y repite los pasos 2 y 3 .

5 Anota los datos en la tabla de abajo. Analiza y saca conclusiones.

6 Comunica ideas. Resume tus resultados.

7 Infiere. ¿Dónde podrías encontrar un arroyo que fluyera a un ángulo de $55^{\circ}$ ? ¿Dónde podrías hallar un arroyo que fluyera a un ángulo de $15^{\circ}$ ? (MINEDUC, 2012C, p. 184)

La actividad presentada en la cita anterior muestra a la ciencia o al proceso de investigación científica como un método riguroso e inalterable, ajeno a la realidad de cualquier individuo.

$\mathrm{Al}$ igual que en los textos anteriores, en los manuales para el estudiante de quinto y sexto año básico, se propone una serie de actividades en donde la investigación se asume como el cumplimiento de un recetario para alcanzar la verdad. Véase la siguiente cita representativa:

Investigación científica Presencia de vitamina $\mathrm{C}$ en los alimentos

Materiales: -Agua -Un limón -Pastillas de vitamina C -Una caja pequeña de jugo de manzana -Yodo diluido -Tres vasos de plástico pequeños -Tres palos de helado -Una cuchara sopera -Un pedazo de tela. 


\section{Observar y preguntar}

A partir de la información anterior, elabora una pregunta de investigación para determinar la presencia de vitamina $\mathrm{C}$ en los alimentos.

\section{Planificar e investigar}

Primero, diluyan el yodo. Para esto, coloquen 1 gota de yodo en 9 gotas de agua. Luego, reúnan los demás materiales y realicen los siguientes pasos: 1 . Con la cuchara y el pedazo de tela, muelan las pastillas de vitamina C. 2. Coloquen las pastillas picadas en uno de los vasos y agreguen una cucharada sopera de agua. Agiten con el palo de helado y rotulen este vaso como no 1.3 . Expriman el jugo del limón y colóquenlo en otro vaso. Rotulen este vaso como no 2.4 . Coloquen una cucharada sopera de jugo de manzana en el tercer vaso. Marquen este último vaso como no 3 . 5. Agreguen a cada vaso una gota de la dilución de yodo y observen. Completen en sus cuadernos la tabla que aparece en la siguiente página.

\section{Analizar la evidencia y comunicar.}

Luego de completar la tabla y analizar los resultados, respondan las siguientes preguntas en sus cuadernos: a. ¿A qué crees que se debe la coloración que se obtuvo en los tres vasos? b. ¿Cuál es el indicador de la presencia de vitamina $\mathrm{C}$ en el experimento? c. De acuerdo con la pregunta anterior, ¿cuál sería el indicador para la ausencia de vitamina $\mathrm{C}$ ? ¿Cómo lo saben? d. Si hubiesen tomado líquido de cocción de carne para este experimento, ¿habrían detectado vitamina C?, ¿cómo explicarían esta situación? e. Finalmente, realicen un poster que resuma las principales etapas de este experimento. Para esto, lean el anexo 2 de la página 203 del texto. Compartan sus resultados con los demás grupos y con su profesor. (MINEDUC, 2013a, p. 9o) Debe hacerse la salvedad que en cada uno de los textos analizados se propone a los estudiantes el investigar. Variadas son las actividades que llevan por título "investiga", pero al ser analizadas se evidencia que las acciones posteriores y el concepto en sí mismo, está siendo comprendido como sinónimo de buscar información en la web o en diferentes medios escritos. Esto se evidencia en las siguientes citas, extraídas del texto de tercer año básico:

Investiga. Observa el siguiente mapa de Chile. En él se han indicado algunos de los principales cultivos de la zona norte, centro y sur de nuestro país. Te invitamos a identificar en qué zona vives tú e investigar y completar el mapa con otros cultivos que se generen en tu zona. (MINEDUC, 2012b, p. 52)

Investiga. Busca en libros o Internet información sobre otras plantas medicinales. Elabora un afiche sobre sus propiedades curativas y expónselo a tus compañeros de clase. (MINEDUC, 2012b, p. 54) 
A partir de lo presentado en cada uno de los textos de estudio para esta categoría de análisis, se puede indicar que existe una distinción entre quienes construyen los conceptos científicos y quienes los estudian en las escuelas.

Esta ciencia escolar a su vez incidiría en la representación social que se construye de la ciencia, mostrándola como una agencia externa a la realidad misma de las personas, representando una ciencia acabada y resuelta, ya que se presentan elementos ya definidos, sin ahondar luego en la elaboración de los mismos.

\section{Conclusiones generales y prospectiva}

\subsection{Síntesis y discusión de los resultados de la investigación}

Con todo lo antes mencionado, se puede indicar que existen dos grupos de categorías, uno de ellos promueve una representación de ciencia en acción, vinculado con los nodos reflexividad y prácticas propias del quehacer científico, y un segundo grupo que promueve una representación de ciencia elaborada, vinculado con las categorías prácticas ingenuas y prácticas que promueven saberes ya elaborados o dentro de una caja negra.

Los manuales analizados plasman un discurso oficial que contribuye en la construcción de determinadas representaciones sociales, en las que los conceptos o hechos científicos se encuentran en el vacío, se producen sin saber cómo, dónde ni por qué, además de proponer que las prácticas científicas las realizan especialistas, quedando a los inexpertos la mera repetición de los procesos ya establecidos.

De acuerdo con lo observado se podría considerar que los textos de estudio y de apoyo a la enseñanza de las ciencias naturales están centrados en transmitir y construir una representación social más bien vinculada con la ciencia elaborada. Constituyéndose esta representación como el saber de sentido común más potente dentro de los textos.

\subsection{Conclusiones}

En el caso particular de este estudio se pudo ahondar en las representaciones que se hacen de las prácticas científicas y de la ciencia en general, reconociendo así la visión oficial que se pretende transmitir del conocimiento científico. 
Se ha podido evidenciar que las prácticas presentadas, aunque varían tanto por curso como por unidad, tienden a producir y reproducir representaciones asociadas a una ciencia elaborada, ya que se presentan prácticas propias de una ciencia absoluta, con saberes imposibles de ser modificados, encerrados dentro de una gran caja negra (Latour, 1992), careciendo muchas veces de contexto y de historia. Los estudiantes deben corroborar saberes previamente establecidos, por lo tanto, se transmite que la ciencia es meramente comprobatoria y no explicativa.

Como plantea Ledesma (2005), se presenta una ciencia ingenua, ciencia que no se constituye como un proceso creativo y humano. La mayoría, por no decir la totalidad de las acciones, se encuentra decretando que para hacer "investigaciones" se deben seguir pasos de carácter inexorable; cualquier error podría traducirse como falta de rigurosidad en el trabajo científico, lo que a la larga produciría problemas sustanciales en los resultados del proceso comprobatorio investigativo.

El tipo de prácticas presentadas y requeridas en las primeras etapas del desarrollo educativo de los sujetos, podría influir en la construcción que se hace de la ciencia en la comunidad y en la ciudadanía en general. Como se mencionó anteriormente, es en esta etapa del desarrollo humano en donde se afianzan y fabrican las ideas de mundo, por lo que tendría un alto valor la manera en la que se enseña ciencias naturales en las escuelas primarias o de enseñanza básica.

Muchas de las acciones, representadas en preguntas, permiten analizar y comprender ciertas propiedades y características de los fenómenos, se posibilita su caracterización así como su aplicación en aspectos de la vida cotidiana. Pero no se dan las condiciones para reflexionar o experimentar las circunstancias en las que dicho fenómeno o conocimiento científico se fundó y construyó.

Si las acciones propias del quehacer científico propuestas en los textos de estudio aumentaran de manera considerable, y si además se aplicaran de manera correcta (Vergara, 2006), se podría contribuir en la construcción de una representación social de una ciencia más bien construida y comprensiva. En palabras de Latour (1992), de una ciencia en acción, porque es a través de las experiencias vividas, en este caso experiencias de investigación, que se participa y propicia una construcción más real de los procesos de investigación científica (Campanario, 1999; Hofstein \& MamlokNaaman, 2007). Los estudiantes, al realizar investigaciones, reconocen que éstas se producen bajo circunstancias especiales, así como también que se encuentran determinadas por las características de quien las desarrolla. Con esto reconocerían que los conceptos tratados, así como los investigadores participantes, se constituyen como 
partes de una historia, historia que durante todo el proceso de construcción transforma a quien investiga así como a lo que se investiga (Latour, 2001).

Con esto no sería necesario estar develando o abriendo cada una de las cajas negras en las que los conocimientos científicos se presentan, ya que cada estudiante contaría con las herramientas para comprender los procesos de construcción del conocimiento científico.

Esta ciencia elaborada transmitida por los textos, ajena a los estudiantes, puede y debe ser desarrollada por especialistas, ya que sólo ellos poseen las llaves maestras para abrir y develar sus secretos.

Los saberes, prácticas, ideas y valores que se están promoviendo, representan una ciencia que permite comprender los hechos y fenómenos de manera externa, no se promueve la comprensión de sus partes ni menos aún de sus orígenes.

\section{Lista de referencias}

Bunge, M. (1960). La Ciencia. Su método y su filosofía. Buenos Aires: Siglo Veinte.

Campanario, J. (1999). La ciencia que no enseñamos. Enseñanza de las Ciencias: Revista de Investigación y Experiencias Didácticas, 17(3), 397-410. Recuperado de https://www.raco.cat/index.php/Ensenanza/article/view/21592

Cofré, H., Camacho, J., Galaz, A., Jiménez, J., Santibáñez, D., \& Vergara, C. (2010). La Educación Científica en Chile: debilidades de la enseñanza y futuros desafíos de la educación de profesores de ciencia. Estudios Pedagógicos, 36(2), 279-293.

Devés, R. (2007). Principios y estrategias del programa de educación en ciencias basada en la indagación. Revista Pensamiento Educativo, 41(2), 115-13. Recuperado de http://pensamientoeducativo.uc.cl/index.php/pel/article/view/419

Domínguez, S. (2009). Las representaciones sociales de la ciencia en alumnos de pregrado del Centro Universitario de Ciencias de la Salud (cucs), Universidad de Guadalajara (Tesis de doctorado inédita). Universidad de Guadalajara, México.

Driver, R., Newton, P., \& Osborne, J. (200o). Estableshing the norms of scientific argumentation in classrooms. Science Education, 84(3), 287-312.

Gunter, E. G. (2002). Las representaciones sociales. Recuperado de http://www.catedras.fsoc.uba.ar/ferraros/BD/egg\%2olas\%2orepresentaciones.pdf

Harlen, W. (s.f.). Aprendizaje y enseñanza de ciencias basados en la indagación. Recu- 
perado de https://gfisicaolaya.files.wordpress.com/2010/og/aprendizaje-yensencc83anza-de-ciencias-basados-en-la-indagaciocc81n.pdf

Haury, D. (1993). Teaching Science through Inquiry. ERIC Clearinghouse for Science Mathematics and Environmental Education Columbus $\mathrm{OH}$. Recuperado de https://files.eric.ed.gov/fulltext/ED359048.pdf

Hofstein, A., \& Mamlok-Naaman, R. (2007). The laboratory in science education: the state of the art. Chemistry Education Research and Practice, 8(2), 105-107. Recuperado de https://pubs.rsc.org/en/journals/journalissues/rp\#!lissueid=rpoo8 $002 \&$ type $=$ current\&issnprint $=1109-4028$

Holbrook, J., \& Raminknae, M. (2007). The nature of science education for enhancing scientific literacy. International Journal of Science Education, 29(11), 13471362. doi:10.1080/09500690601007549

Hughes, G. (2000). Marginalization of socioscientific material in Science-Technology-Society Science Curricula: Some implications for gender inclusivity and curriculum reform. Journal of Research in Science Teaching, 37(5), 426-440.

Latour, B. (1983). Give Me a Laboratory and I will Raise the World. En K. KnorrCetina \& M. Mulkay (Eds.), Science Observed: Perspectives on the Social Study of Science (pp. 141 -170). Londres: Sage.

Latour, B. (1992). Ciencia en acción. Barcelona: Labor.

Latour, B. (2001). La Esperanza de Pandora. Barcelona: Gedisa.

Ledesma, I. (2005). De Kuhn a Latour: La investigación, la ciencia y los estudios sociales de la ciencia. Erinias, $1(3), 77-85$.

Ministerio de Educación. (2012a). Ciencias Naturales. Primero básico. Texto del estudiante. Santiago, Chile: Santillana.

Ministerio de Educación. (2012b). Ciencias Naturales. Tercero básico. Texto del estudiante. Santiago, Chile: Pearson Educación de Chile Ltda.

Ministerio de Educación. (2012c). Ciencias Naturales. Cuarto básico. Texto del estudiante. Santiago, Chile: Pearson Educación de Chile Ltda.

Ministerio de Educación. (2013a). Ciencias Naturales. Quinto básico. Texto del estudiante. Santiago, Chile: Santillana.

Ministerio de Educación. (2013b). Ciencias Naturales. Segundo básico. Texto del estudiante. Santiago, Chile: Santillana.

Ministerio de Educación. (2013c). Ciencias Naturales. Sexto básico. Texto del estudiante. Santiago, Chile: Santillana.

Moscovici, S. (1984). The phenomenon of social representations. En R. Farr \& S. 
Moscovici (Eds.), Social Representations. Cambridge, Inglaterra: Cambridge University Press.

Moscovici, S. (1988). Social Representations. Notes towards a description of social representations. European Journal of Social Psychology, 18, 211-50.

Moscovici, S. (2000). Social Representations. Cambridge: Polity.

Organización de las Naciones Unidas para la Educación, la Ciencia y la Cultura. (2000). Marco de acción de Dakar. Educación para Todos: cumplir nuestros compromisos comunes (con los seis marcos de acción regionales). Foro Mundial Sobre la Educación. Dakar, Senegal: Organización de las Naciones Unidas para la Educación, la Ciencia y la Cultura.

Pugh, K. (2009). Motivation, learning and transformative experience: A study of deep engagement in science. Science Education, 94(1), 1-28.

Vergara, C. (2006). Concepciones sobre la enseñanza y el aprendizaje en profesores de biología: Coherencia entre el discurso y la práctica de aula (Tesis doctoral inédita). Pontificia Universidad Católica de Chile, Chile.

Yáñez, C. (2010). Representaciones sociales de las identidades en los textos escolares de ciencias sociales en Colombia. Cadernos de Educação, 37, 15-38. Recuperado de https://periodicos.ufpel.edu.br/ojs2/index.php/caduc/article/view/1578

Ziman, J. (2003). ¿Qué es la ciencia? (Trads. E. Pérez \& N. Galicia). Cambridge: Cambridge University Press. 